\title{
Dengue, bleeding and non-steroidal anti-inflammatory drugs
}

\author{
Wijewickrama $\mathbf{A}^{1}$
}

Journal of the Ceylon College of Physicians, 2017, 48, 66-74

\section{Introduction}

Dengue is the most rapidly spreading mosquitoborne viral disease in the world ${ }^{1}$. It is estimated that between 50 and 100 million cases of DF and several hundred thousand cases of DHF occur each year, depending on the epidemic activity².

In the last 50 years, incidence has increased 30 fold with increasing geographic expansion to new countries and from urban to rural settings ${ }^{1}$. More than 100 tropical countries have endemic Dengue virus infections. This includes most of Asian countries ${ }^{2}$ including Sri Lanka.

\section{Situation in Sri Lanka}

Dengue, which was serologically first confirmed in 1962 in Sri Lanka, has become endemic since $1989^{3}$. The magnitude of Dengue epidemics in Sri Lanka has continued to increase stepwise with regular epidemics ${ }^{4}$ and currently it has become a major health hazard in with a high morbidity 5 . Since 2009 , the annual incidence rate has been more than 150 per 100,000 population $^{6}$. In 2017, this increased to very high levels recording 75,000 cases within the first 06 months of the year.

\section{Dengue classifications}

The widely used classification of Dengue, introduced by the WHO in 1997, divided Dengue into two main groups i.e. Dengue Fever (DF) and Dengue Haemorrhagic Fever (DHF). However, another classification was proposed by the WHO in 2009, (usually called WHO/TDR classification) where it was divided into three categories:

1. Dengue without warning signs

2. Dengue with warning signs

3. Severe dengue

The need for reclassification was due to the strict nature of the definition of the DHF, where 04 essential criteria need to be fulfilled. We showed in a study of 106 clinically diagnosed Dengue patients, published in the Journal of Ceylon College of Physicians in 2011, of 28 clinical DHF cases only five (17.9\%) fulfilled all four WHO criteria (1997) for DHF, whilst $23(82.1 \%)$ did not fulfil all four criteria though they developed detectable effusions/ascites ${ }^{7}$. The new WHO classification of 2009 introduced the concept of warning signs. Our study showed that this is very useful as all but one patient with DHF had at least one warning sign (Table 1).

Table 1. Prevalence of WHO prescribed warning sign in clinical DF and DHF patients

\begin{tabular}{lcccc}
\hline WHO warning sign & $\begin{array}{c}\text { Clinical DF } \\
(n=78)\end{array}$ & $\begin{array}{c}\text { Clinical DHF } \\
(n=28)\end{array}$ & $\begin{array}{c}\text { Total } \\
(n=106)\end{array}$ & P value \\
\hline Increased haematocrit & $36(46.2 \%)$ & $20(71 \%)$ & $58(54.7 \%)$ & $<0.05$ \\
Abd. Pain/tenderness & $47(60.3 \%)$ & $18(64.3 \%)$ & $65(61.3 \%)$ & $>0.05$ \\
Persistent vomiting & $6(7.7 \%)$ & $3(10.7 \%)$ & $8(7.5 \%)$ & $>0.05$ \\
Mucosal bleeding & $3(3.8 \%)$ & $5(17.9 \%)$ & $8(7.5 \%)$ & $<0.05$ \\
Fluid accumulation & $0(0 \%)$ & $7(25 \%)$ & $7(6.6 \%)$ & $<0.05$ \\
Restlessness & 0 & $1(3.6 \%)$ & $1(0.9 \%)$ & $>0.05$ \\
Hepatomegaly>2cm & 0 & $8(28.6 \%)$ & $8(7.5 \%)$ & $<0.05$ \\
\hline
\end{tabular}

${ }^{1}$ Consultant Physician, National Institute of Infectious Diseases, Colombo, Sri Lanka.

This is an open-access article distributed under the terms of the Creative Commons Attribution License, which permits unrestricted use, distribution, and reproduction in any medium, provided the original author and source are credited. 
However, the new WHO/TDR classification of $2009^{1}$ had its own problems. Our analysis, published in the article "Usefulness of World Health Organization (WHO) dengue case classifications in a Sri Lankan clinical setting", which is shown in Table 2, demonstrated how $76.9 \%$ of DF patients were grouped together with $40 \%$ of DHF 3 \& 4 patients 7 .

According to the WHO/TDR classification, patients with warning signs require strict observation. Since these patients form the majority, compliance with the WHO TDR guidance would probably overburden clinical facilities. We pointed this out in an article written to WHO South-East Asia Journal of Public Health in $2014^{8}$.

It was in this backdrop, in 2011, WHO SEARO group modified the 1997 criteria. In fact, it is an improvement and the main changes were the diagnosis of plasma leakage by evidence of pleural effusion and/ or ascites and the inclusion of two groups, namely, unusual manifestations and Dengue with bleeding.

In "Expediency of dengue illness classification: the Sri Lankan perspective" published in WHO SouthEast Asia Journal of Public Health in $2014^{8}$ we pointed out that the 1997/2011 WHO classification identifies DF and DHF as two distinct entities; by contrast, the 2009/12 WHO/TDR classification considers that "dengue is one disease entity with different clinical presentations and often with unpredictable clinical evolution and outcome" ${ }^{8}$. We further pointed out that the major advantage of the 1997/2011 WHO classification, which has helped to guide clinicians to manage patients without allowing complications of severe dengue to arise, making DHF a "predictably treatable illness" is the fact that concurrent rise in haematocrit is recommended to differentiate DF from $\mathrm{DHF}^{8}$.

Therefore, we find, WHO/SEARO 2011 classifi- cation a very user friendly one and we have recommended its use in our own clinical guideline.

\section{Changes in the virus serotype}

Dengue virus has four serotypes. While all were found to be present in Sri Lanka, predominant serotype varied from time to time. However, analysis of serotypes in early 2016 and early 2017 showed a drastic change in circulating serotype. Analysis of 75 randomly selected patients admitted to National Institute of Infectious Diseases in January 2016 showed 92\% had serotype 1 whereas a similar analysis done in January 2017 of 48 patients showed complete replacement of serotypes by Dengue serotype 2 . We postulate that this is one of the reasons for the massive outbreak of Dengue we are experiencing at present (2017) in Sri Lanka.

\section{Demography}

In South-East Asian countries, where all the serotypes (DENV-1-4) are circulating, DF was typically acknowledged to be a disease of early childhood, while clinical DF in adults was rare. However, there is evidence of increase of dengue incidence in older age groups, and this age shift has been reported in Singapore, Indonesia, Bangladesh and Thailand'.

Similarly, in Sri Lanka also Dengue had been predominantly a paediatric illness. In 80 's and early 90's the age distribution pattern of Dengue patients in Sri Lanka showed that two thirds of cases were children under 15 years with a peak incidence in the 5-9-year age group ${ }^{3}$. However, even then, a significant number of cases were noted in the 15-29-year age group, especially between $15-19$ years $^{3}$. By now, the modal age group affected by dengue has shifted from $<15$ years of age to 15-34 years of age ${ }^{9}$, with more than $75 \%$ of cases occurring in the age group above 15 year of age $\mathrm{e}^{6}$.

Table 2. Comparison of warning signs between the two WHO classifications

\begin{tabular}{lcccc}
\hline & $\begin{array}{c}\text { Dengue without } \\
\text { warning signs }\end{array}$ & $\begin{array}{c}\text { Dengue with } \\
\text { warning signs }\end{array}$ & Severe Dengue & Total \\
\hline DF & $18(23.1 \%)$ & $60(76.9 \%)$ & 00 & 78 (73.6\% of all $)$ \\
DHF 1 \& 2 & 00 & $18(100 \%)$ & 00 & $18(17 \%$ of all $)$ \\
DHF 3 \& 4 & $1(10 \%)$ & $4(40 \%)$ & $5(50 \%)$ & 10 (9.4\% of all) \\
Total & $19(17.95)$ & $82(77.4 \%)$ & $5(4.7 \%)$ & 106 \\
\hline
\end{tabular}




\section{Differences in children and adults with Dengue}

Symptoms and risk factors for Dengue haemorrhagic fever (DHF) and severe Dengue differ between children and adults. In a three year study, where the severity of Dengue was investigated in infants, children and adults, it was found that frequency of internal bleeding was significantly higher in adults compared to other two groups ${ }^{10}$. In a study of Dengue deaths in a Malaysian hospital, $80 \%$ of deaths were among adults ${ }^{11}$. Another prospective descriptive study of 947 children and 738 adults in Vietnam, found that plasma leakage and shock were more common and severe in children than in adults, while bleeding and organ dysfunction were more frequent in adults ${ }^{12}$. In the 2001 epidemic in Chonburi, Thailand, clinical bleeding was significantly more frequent in adults ${ }^{13}$. In a large retrospective study done in Singapore, 1,035 (14.8\%) adults had severe dengue according to WHO 2009 criteria and of these, $40 \%$ had severe bleeding ${ }^{14}$.

Bleeding is a frequent cause of severe dengue illness, especially in adults ${ }^{15}$. Petechiae, epistaxis and menorrhagia have been observed frequently in adults with DF or DHF, although upper gastro-intestinal (Gl) bleeding is the most common type of severe haemorrhage. Menorrhagia is common in female adults with DF/DHF. Bleeding into sites such as subcapsular region of spleen and splenic rupture have also been reported in adults with dengue infection ${ }^{16}$ and such bleeding can be occult. A Taiwanese study showed that massive gastrointestinal bleeding accounted for $40 \%$ of dengue haemorrhagic fatalities ${ }^{17}$ and similar findings were found in a mortality study in Singapore ${ }^{18}$.

Severe but occult bleeding can be difficult to recognize. Many patients with severe bleeding have initial or on-going plasma leakage that keeps the HCT in the normal range despite ongoing bleeding ${ }^{15}$. Therefore, haematocrit may not be a sensitive marker of plasma leakage in dengue with severe bleeding ${ }^{11,15}$.

The ability to identify patients at high risk of progression to severe disease, who are likely to benefit from close observation and early intervention with supportive therapy, has become the focus of intense research efforts in recent years ${ }^{19}$. This has become important as the vast majority of symptomatic infections do not progress to severe disease, but as this is unpredictable, monitoring of large numbers of patients in seasonal epidemics overwhelms health service capacity in many Dengue prevalent areas. Most studies have focused on paediatric patients. Data for adult Dengue patients are sparse. In addition, most studies used are expensive, advanced laboratory methods, which are not suitable for limited resource settings ${ }^{21}$.

Several retrospective studies were done in attempting to identify the predictors of bleeding in adults with Dengue. However, prospective studies are few and involved only a small number of patients ${ }^{20,22}$. Hence we conducted a prospective study to determine clinical parameters which could be easily used to identify Dengue patients who are likely to develop bleeding. Results of this study were presented at the Annual Conference of the American Society of Tropical Medicine and Hygiene in 2016. In this study all patients admitted to Dengue Management Unit at the National Institute of Infectious Diseases, Colombo for four months from 1st of July 2014 were included. Dengue was confirmed by NS1 antigen or Dengue Specific IgM antibodies. These patients were followed up to see the development of bleeding, possible effects of bleeding and the need of blood transfusion.

There were 1000 patients with confirmed Dengue infection (546 males and 454 females). Age ranged from 12 to 86 years. (Mean 31). 562 had DF (56.2\%); $438(43.8 \%)$ had DHF. 332 (33.2\%) had some degree of bleeding; major bleeding in $17.0 \%$, minor bleeding in $15.67 .1 \%$ had no bleeding at all, other than petechial bleeding. 81 (8.1\%) needed therapeutic blood transfusions.

Association of various parameters with bleeding is shown in Table 3.

Our study showed female gender and obesity $(\mathrm{BMI}>27.5)$ are characteristics which are significantly associated with bleeding. Female sex has been identified as a risk factor for bleeding in other studies as well ${ }^{23}$. Though it is an established fact that obese patients are more prone to develop plasma leakage, association of bleeding with obesity has not been described before. Severe or persisting vomiting, abdominal pain, postural dizziness and use of NSAID during the illness were the associated significantly with bleeding. Vomiting and abdominal pain ${ }^{24}$ were identified as predictors of bleeding in previous studies, but postural dizziness and NSAID use were not. Postural dizziness would indicate the intravascular volume depletion and should alert the medical staff. NSAID use is an avoidable risk factor. Hence the identification of these is very important and useful.

In Sri Lanka, the National Guidelines on management of Dengue Fever and Dengue Hemorrhagic Fever recommends against using Non-Steroidal-Anti Inflammatory-Drugs (NSAID) in patients with Dengue 
Table 3. Predictors of bleeding

\begin{tabular}{|c|c|c|c|c|c|c|c|}
\hline & & $\begin{array}{c}\text { Major } \\
\text { bleeding }\end{array}$ & Other & $P$ value & $\begin{array}{l}\text { Odds } \\
\text { ratio }\end{array}$ & & $\begin{array}{l}\text { dence } \\
\text { rval }\end{array}$ \\
\hline & & & & & & Lower & Upper \\
\hline Severe vomiting & $\begin{array}{l}\text { Yes } \\
\text { No }\end{array}$ & $\begin{array}{c}36(15.3 \%) \\
200(84.7 \%)\end{array}$ & $\begin{array}{c}67(9 \%) \\
674(91 \%)\end{array}$ & $<0.05$ & 1.811 & 1.172 & 2.797 \\
\hline Postural dizziness & Yes & $151(64 \%)$ & 489 (52.6\%) & $<0.05$ & 1.603 & 1.185 & 2.169 \\
\hline & No & 85 (36\%) & 351 (47.4\%) & & & & \\
\hline NSAIDs use & Used & $22(9.3)$ & $41(5.5 \%)$ & $<0.05$ & 1.755 & 1.023 & 3.012 \\
\hline & & & & & & & \\
\hline Sex & Male & $74(31.4 \%)$ & $462(62.3 \%)$ & $<0.05$ & 3.625 & 2.652 & 4.955 \\
\hline & Female & $162(68.6 \%)$ & $279(37.7 \%)$ & & & & \\
\hline $\mathrm{BMI}<23 \&>23$ & $<23$ & $134(57 \%)$ & 436 (58.8\%) & $>0.05$ & 1.077 & 0.801 & 1.450 \\
\hline & $>23$ & $101(43 \%)$ & $305(41.2 \%)$ & & & & \\
\hline $\mathrm{BMI}<27.5 \&>27.5$ & $<27.5$ & 197 (83.8\%) & 659 (88.9\%) & $<0.05$ & 1.550 & 1.022 & 2.351 \\
\hline & $>27.5$ & 38 (16.2\%) & $82(11.1 \%)$ & & & & \\
\hline Plt $<50000 \&>50000$ & $<50000$ & 187 (79.2\%) & $512(69.1 \%)$ & $<0.05$ & 1.707 & 1.202 & 2.425 \\
\hline & $>50000$ & 49 (20.8\%) & 229 (30.9\%) & & & & \\
\hline Hepatic tenderness & Yes & $85(36 \%)$ & $171(23.1 \%)$ & $<0.05$ & 1.876 & 1.368 & 2.524 \\
\hline & No & $151(64 \%)$ & $570(76.9 \%)$ & & & & \\
\hline Abdominal pain & Yes & $94(39.8 \%)$ & $226(30.5 \%)$ & $<0.05$ & 1.506 & 1.111 & 2.040 \\
\hline & No & $142(60.2 \%)$ & $514(69.5 \%)$ & & & & \\
\hline Restlessness & Yes & $3(1.3 \%)$ & $4(0.5 \%)$ & $>0.05$ & 2.372 & 0.527 & 10.676 \\
\hline & No & 233 (98.7\%) & 737 (99.5\%) & & & & \\
\hline PCV drop & PCV drop & $2(0.8 \%)$ & $7(0.9 \%)$ & $>0.05$ & 1.116 & 0.230 & 5.408 \\
\hline & No PCV drop & 234 (99.2\%) & 734 (99.1\%) & & & & \\
\hline Retro orbital pain & Yes & $83(35.2 \%)$ & $223(30.1 \%)$ & $>0.05$ & 0.794 & 0.582 & 1.082 \\
\hline & No & $153(64.8 \%)$ & $518(69.9 \%)$ & & & & \\
\hline Myalgia & Yes & $214(90.7 \%)$ & $671(90.6 \%)$ & $>0.05$ & 0.958 & 0.596 & 1.630 \\
\hline & No & $22(9.3 \%)$ & 70 (9.4\%) & & & & \\
\hline Headache & Yes & 217 (91.9\%) & 673 (90.9\%) & $>0.05$ & 0.879 & 0.517 & 1.497 \\
\hline & No & $19(8.1 \%)$ & $67(9.1 \%)$ & & & & \\
\hline Arthralgia & Yes & 186 (78.8\%) & 548 (74.0\%) & $>0.05$ & 0.763 & 0.536 & 1.086 \\
\hline & No & $50(21.2 \%)$ & $193(26.0 \%)$ & & & & \\
\hline
\end{tabular}

infection ${ }^{25}$. Similar recommendations are made in other Dengue management guidelines ${ }^{1,26}$. However, there is no clinical evidence for these recommendations and they are made based on expert opinion.

NSAIDs are prescribed by some general practitioners (GPs) for symptomatic treatment of severe body aches and high fever in many patients with fever, before a proper diagnosis is made, of whom many could be having Dengue in an endemic setting. Unfortunately, symptoms of Dengue are similar to any other viral fever at the initial stage ${ }^{4,5}$. Furthermore, these drugs are 
available 'over the counter', and therefore the use of NSAIDs as a remedy for fever and body aches has become a practice among the general population.

The gastrointestinal consequences of nonsteroidal anti-inflammatory drugs (NSAIDs) are the best recognized iatrogenic problem in clinical medicine ${ }^{27}$. In the majority of patients, NSAID-induced gastroduodenal mucosal injury is superficial and self-limited. However, peptic ulcers develop in some patients, and they may lead to gastro duodenal hemorrhage, perforation, and death ${ }^{28}$. In addition, NSAID can cause damage to the more distal part of the small intestine and there are many case reports suggesting that they can affect the large intestine causing colitis and colonic perforation $^{28,29}$. It is estimated to cause 3500 hospitalizations and 400 deaths in 1.5 million of NSAID taking population of people above the age of 60 years in the United Kingdom ${ }^{29}$. There is clear evidence that severe complications associated with peptic ulcer disease are often associated with recent NSAID consumption $^{29}$. While the concomitant use of normal doses of $\mathrm{H}-2$ receptor blockers do not effectively prevent NSAID induced peptic ulcer disease, Proton Pump Inhibitors are unlikely to reduce lower GI complications ${ }^{27}$. Although 'serious systemic illnesses' is listed as one of the risk factors for the development of NSAID induced peptic ulcers, ${ }^{28}$ Dengue illness has not been identified as one.

Hepatotoxicity is another uncommon, but potentially lethal complication of NSAID and it can occur with all NSAIDs. They exhibit a broad spectrum of liver damage ranging from asymptomatic, transient, hypertransaminasemia to fulminant hepatic failure ${ }^{30}$. However, the risk of developing acute liver injury is very low in epidemiological studies though diclofenac is known to have an excess risk ${ }^{31-33}$. Though hepatotoxicity usually occurs 12 weeks after initiation of therapy, it can occur at any time after drug administration ${ }^{30}$. Several risk factors have been identified for people to be more prone to develop NSAID induced hepatotoxicity but relationship with Dengue has not been assessed.

Therefore, it is prudent to determine the actual role of NSAID, on bleeding manifestations and liver damage in Dengue infection. This particular study represents, to our knowledge, the first study done on effects of NSAIDS on bleeding and liver in Dengue infection. A careful literature search on Medscape did not reveal any previous studies done on this subject. Even the WHO guidelines on management of Dengue does not give any supporting evidence for its recommendation of not to use NSAIDS in Dengue infection. Findings of this study were presented at the 17th International Congress on Infectious Diseases (ICID) in 2016 and was awarded the ProMED award.

A prospective 'Case-control' Study design was selected for this study as a 'Randomized controlled trial' could raise ethical issues since treatment with NSAIDs is virtually not recommended, even though there is no evidence for the recommendation.

The study population comprised of Dengue patients aged above 12 years, admitted to the hospital during 1 stJune 2014 to 30th September 2014. Patients who were admitted to the Dengue Management Unit (DMU) with fever of acute onset with myalgia and headache and confirmed as Dengue infection were included in the study. Dengue was confirmed either by positive NS1 antigen or Dengue specific IgM antibodies or both. Patients who were on aspirin, clopidogrel or heparin were excluded since those drugs are known to increase risk of bleeding manifestations.

Patients who had been treated (or self-treated) with NSAID during the febrile phase before admission to hospital were taken as cases and those who did not have NSAID were taken as controls. Data was collected using an interviewer administered questionnaire. Patients were questioned about the medicines they took prior to admission to the hospital. The prescriptions given by the General Practitioners were reviewed and the medicines the patients brought were inspected. If there was definitive evidence to of NSAID intake they were categorized as 'cases' and if there was definitive evidence of not taking NSAIDS they were categorized as 'controls'. When it was uncertain whether the patients have taken NSAID or not, they were not taken in to study analysis.

NS1 and/or Dengue IgM Antibody test were done in all patients. All patients were managed in a single unit according to the National Guidelines of Sri Lanka and therefore, the management was uniform. The patients were regularly reviewed with monitoring of vital signs. Full blood counts were done daily and haematocrit was done 6 hourly. Regular ultra sound scans of the chest and abdomen were done to detect plasma leakage. If the plasma leakage or bleeding was detected monitoring was intensified for the next 48 hours or more. Serum Aspartate transaminase (AST) levels and alanine transaminase (ALT) levels were done daily.

Rise in AST and ALT levels and presence of bleeding manifestations were taken in to consideration in the analysis as those are the commonest recognized effects of NSAID which are relevant to Dengue infection. 
Minor bleeding manifestations such as positive tourniquet test, petechial bleeding, gum bleeding, occasional epistaxis, haemoptysis and haematuria which are common both in DF and DHF were not considered for analysis. Major bleeding such as hypermenorrhea, severe epistaxis, intermenstrual bleeding, haematamesis, malaena, and occult bleeding were considered for analysis. Reduction of haematocrit with unstable vital signs necessitating blood transfusions were taken as evidence of occult bleeding.

\section{Results}

All the patients, who were serologically confirmed as having Dengue, admitted to the Dengue Management Unit (DMU) from 1st of June to 30th of September were prospectively studied. The total study population was 1000 patients with 546 males and 456 females. Age ranged from 12 to 86 years. The mean age was 31 years. $30.1 \%$ were between $21-30$ years of age while $28.3 \%$ were between $12-20$ years. None were on warfarin, heparin or clopidogrel. In a random sample of 18 cases 15 were due to DENV 1 serotype infection and 2 were due to DENV 4 serotype. ( 1 patient could not be serotyped). 562 (56.2\%) had DF; 438 (43.8\%) had DHF. Higher number of DHF was due to the fact that DF patients were admitted to other wards and only those who needed close monitoring were admitted to Dengue Monitoring Unit.

Out of 1000 patients, $6.5 \%(n=65)$ have been definitely treated with NSAIDs prior to admission, while $57.7 \%(n=577)$ have not used NSAIDs definitely; the rest $(35.8 \%)$ were not certain. Mean age of NSAID group was 33.57 years (SD 17.124) while it was 31.34 years (SD 14.419) in the non-NSAID (control) group. Male: female ratio was $1: 1$ in the NSAID group while it was 1:0.92 in the non-NSAID group.

Major bleeding occurred in $44.6 \%$ of patients who had NSAID and in $30.32 \%$ of patients who did not have NSAID. ( $<<0.024$, Odds ratio: 1.818 (1.075 - 3.076). When this was separately analyzed in DF and DHF groups, results were still significant; Out of DF patients $28 \%$ developed bleeding in the non-NSAIDS group while $36.7 \%$ had bleeding in the NSAIDS group $(p<0.05)$. Among the DHF patients $33.92 \%$ had bleeding in nonNSAIDS group while $51.42 \%$ had bleeding in the NSAIDS group $(p<0.05)$. Higher percentage of NSAID users $(9.2 \%)$ required blood transfusions compared to non-NSAID users $(6.2 \%)$ though this was not statistically significant. NSAID use is associated with a wide array of alterations in gastro intestinal tract integrity and function; among the most common of these are hemorrhagic gastric erosions. It has been also recognized that NSAIDS can damage more distal regions of the small intestine and also the colon ${ }^{29}$. Clinically significant gastric bleeds from erosions is very rare in patients without clotting or platelet impairment ${ }^{31}$. Thrombocytopenia is universal in DHF and also seen in many with DF. Furthermore, platelet function is abnormal in dengue infections with impaired platelet aggregation and markedly shortened platelet survival. In a study of 170 children with Dengue Shock Syndrome abnormalities were seen in all the major pathways of the coagulation cascade (i.e., low levels of the natural anticoagulant proteins and increased levels of the major procoagulant and anti-brinolytic agents), even though serious bleeding manifestations were relatively infrequent ${ }^{32}$. In this context, it is clear that NSAID can lead to increased bleeding in patients with dengue. Our study confirmed this.

In addition to the effect of bleeding, our study showed the degree of hepatitis, as evident by the rise in liver enzymes, was significantly higher in those who had NSAID.

In our study, $24.6 \%$ of the NSAIDs users and $14.7 \%$ of non-NSAID users had ALT above $300 \mu / \mathrm{L}$ $(p<0.05$ Odds ratio: $2.105(0.883-5.014)) .1 .538 \%$ NSAID users and no non-NSAID users had ALT greater than $1000 \mu /$ L. $36.92 \%$ of NSAID group and $23.74 \%$ of non-NSAID group had AST greater than $300 \mu / \mathrm{L}$. $(p<0.05$. Odds ratio: 2.195 (1.025 - 4.700). 3.07\% of NSAID group and no non-NSAID group had AST greater than $1000 \mu / L$.

In a study of 1585 patients with dengue, the mean elevation of AST and ALT were $93.3 \mathrm{U} / \mathrm{L}$ and $86 \mathrm{U} / \mathrm{L}$ respectively. Only $1.8 \%$ had ALT rise of more than 10 times upper limit of normal (ULN) and 3.4\% had AST rise of more than 10 times ULN. More than 3 times ULN of AST and ALT was seen in $16.1 \%$ and $11.1 \%$ respectively. ${ }^{27}$ Above findings make our study more significant. In the control group of our study $14.8 \%$ had a rise of ALT more than $300 \mathrm{u} / \mathrm{l}$ while $23.8 \%$ had a rise of AST more than $300 \mathrm{u} / \mathrm{l}$. These figures are higher than Souza's study probably because our study had a higher number of DHF patients. However, in NSAID users these were $25.0 \%$ and $37.5 \%$ respectively. This was statistically significant. Furthermore, all patients who had AST rise of more than $1000 \mathrm{u} / \mathrm{l}$ were those who had NSAID. 
This study provides the hitherto unavailable evidence on deleterious effects of NSAID in patients with dengue infection. Dengue patients who had NSAID during the course of the illness had increased incidence of bleeding and also higher elevation of liver enzymes indicating more severe effect on the liver. Since bleeding and liver involvement are two complications which can lead to serious outcomes in dengue, use of NSAID can make patients more liable for worse outcomes. Therefore, we recommend NSAID should not be used in fever patients when dengue is a possibility and in dengue outbreaks NSAID should not be used at all in any fever patient.

Next to blood and blood vessels, liver is the commonest organ to get involved in Dengue. Liver injury itself can give rise to bleeding. Many factors are thought to contribute to liver dysfunction, including hypoxic injury due to decreased perfusion, direct damage by the virus and immune mediated injury. We studied the patterns and causes (other than NSAID) of liver injury in acute dengue infection and the results of this study were published in the journal BMC Infectious Diseases in $2016^{33}$. In this study, we sought to identify the pattern in the change in liver enzymes throughout the illness and its association with the degree of viraemia, onset and extent of plasma leakage and inflammatory mediators.

Serial daily blood samples were obtained from 55 adult patients with acute dengue from the time of admission to discharge and the liver function tests, viral loads and cytokines were assessed. The onset and extent of fluid leakage was measured by daily ultrasound examinations and all clinical and laboratory features were serially recorded.

Results of this study showed aspartate transaminase (AST), alanine transaminase (ALT) and gamma glutamyl transferase (GGT) levels were elevated in patients with dengue infection throughout the illness. The highest AST levels were seen on day 6 of illness and both AST and GGT levels were significantly higher in patients with severe dengue (SD), when compared to those with non-severe dengue (NSD) on day 5 and 6 of illness. Three patients with SD had AST and ALT values of $>1000 / \mathrm{IU}$ in the absence of any fluid leakage or a rise in the haematocrit $(\geq 20 \%)$. The peak of the AST levels and the lowest serum albumin levels were seen $24 \mathrm{~h}$ before the maximum fluid leakage and $24 \mathrm{~h}$ after the peak in viraemia. Both serum IL-10 and IL-17 levels were elevated during early illness and were significantly higher in those with SD when compared to NSD.
The figure below shows how the IL-10 and IL-17 changed over time with the illness:
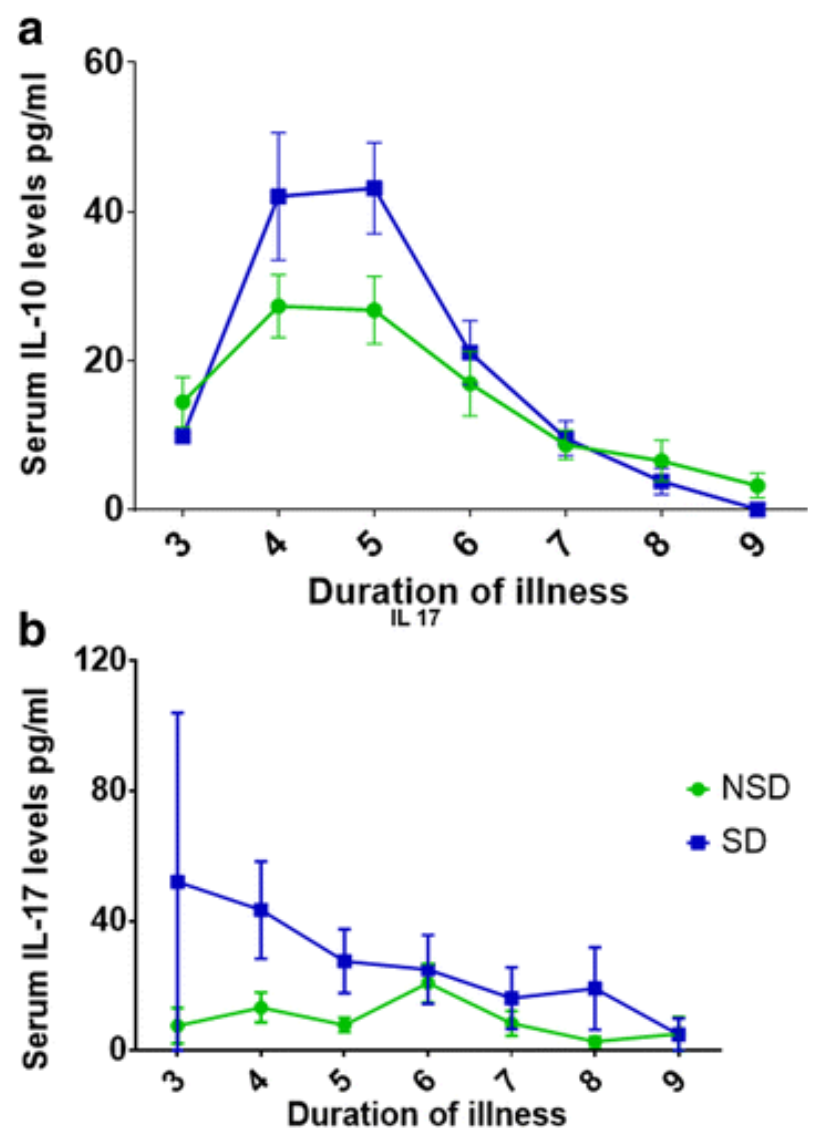

Figure 1. Changes in cytokine levels throughout the course of illness. a: Changes in serum IL-10 levels in patients with SD $(n=22)$ and NSD $(n=33)$. The lines indicate the means and standard error of mean (SEM). b: Changes in serum IL-17 levels in patients with SD $(n=22)$ and NSD $(n=33)$. The lines indicate the means and standard error of mean (SEM).

We found that dengue associated liver injury appears to peak around day 6 and 7. Therefore, liver function tests done at earlier dates might not reflect the extent of liver involvement in acute infection. Since severe liver involvement can occur in the absence of fluid leakage, after the peak viraemia, and since it is associated with high IL-17 and IL-10 levels, possible immune mechanisms leading to hepatic damage is a possibility.

\section{Conclusion}

Dengue has reached epidemic proportions in many tropical countries in the world. A classification which is useful in the clinical management would be 
the most appropriate as it would reduce the burden of hospitals as well as enable clinicians to find patients with complications early. Our studies showed that the DF/DHF classification (WHO 1997/2011) is more useful than the Non Severe Dengue/Severe Dengue (WHO/ TDR 2009) classification.

With changing demography, bleeding associated with Dengue has become commoner. We have identified pre-disposing factors which could be used to identify possible bleeders in dengue. This would enable clinicians to monitor this group of patients more closely so that bleeding can be detected early and necessary interventions can be taken early. We have also shown that liver damage may have an immune mechanism opening doors for further studies in to this area.

More importantly, our studies showed conclusive evidence of the deleterious effects of NSAID in dengue. This would give enough strength to available recommendations to prohibit use of this easily avoidable risk factor.

\section{References}

1. Dengue: Guidelines for treatment, prevention and control. Geneva: World Health Organization, 2009.

2. Gubler DJ. Epidemic dengue/dengue haemorrhagic fever as a public health, social and economic problem in the 21st century. Trends in Microbiology 2002; 10: 2.

3. Vitarana T, Jayakuru WS, Withane N. Historical Account of Dengue Haemorrhagic Fever in Sri Lanka. Dengue Bulletin 1997; 21: 117-118.

4. Kanakaratne, N, Wahala WM, Messer WB, Tissera HA, Shahani A, Abeysinghe N. Gunasekera M. Severe Dengue Epidemics in Sri Lanka, 2003-2006. Emerging Infectious Diseases 2009; 15(2): 192-199.

https://dx.doi.org/10.3201/eid1502.080926.

5. Kularatne SAM, Gawarammana, Kumarasiri PRV. Epidemiology, clinical features, laboratory investigations and early diagnosis of dengue fever in adults: a descriptive study in Sri Lanka. Southeast Asian J Trop Med Public Health 2005; 36 (3): 686-92.

6. Annual Health Bulletin, Sri Lanka - 2014.

7. Wanigasuriya, K, Gurugama P, WijewickremaA, Seneviratne SL, Gunatilake SB. Us efulness of World Health Organization (WHO) dengue case classifications in a Sri Lankan clinical setting. Journal of the Ceylon College of Physicians 2011; 42(1\&2): 21-7.

DOI:http://doi.org/10.4038/jccp.v42i1-2.4550

8. Tissera H, Weeraman J, Amarasinghe A, Wijewickrama A, Palihawadana P, Fernando L. Expediency of dengue illness classification: The Sri Lankan perspective. WHO SouthEast Asia J Public Health 2014; 3: 5-7.
9. Bhatia R, Dash PA, Sunvoto T. Changing epidemiology of dengue in South-East Asia. WHO South-East Asia Journal of Public Health 2013; 2(1):

10. Hammond SN, Balmaseda A, Pérez L. Differences in dengue severity in infants, children, and adults in a 3-year hospital-based study in Nicaragua. Am. J. Trop. Med. Hygiene 2005; 73(6): 1063-70.

11. Sam SS, Omar SFS, Teoh BT, Abd-Jamil J, AbuBakar S. Review of Dengue Haemorrhagic Fever Fatal Cases Seen Among Adults: A Retrospective Study. PLoS Negl Trop Disease 2013; 7(5): e2194. DOI:10.1371/journal.pntd.0002194

12. Dinh The T, Le Thi Thu T, Nguyen Minh D, Tran Van N, Tran Tinh $\mathrm{H}$, et al. Clinical Features of Dengue in a Large Vietnamese Cohort: Intrinsically Lower Platelet Counts and Greater Risk for Bleeding in Adults than Children. PLoS Negl Trop Dis 2012; 6(6): e1679.

DOI:10.1371/journal.pntd.0001679.

13. Wichmann O, Hongsiriwon S, Bowonwatanuwong C, Chotivanich K, Sukthana Y, Pukrittayakamee S. Risk factors and clinical features associated with severe dengue infection in adults and children during the 2001 epidemic in Chonburi, Thailand. Tropical Medicine and International Health 2004; 9 (9): 1022-9.

14. Rowe EK, Leo Y-S, Wong JGX, Thein T-L, Gan VC, et al. Challenges in Dengue Fever in the Elderly: Atypical Presentation and Risk of Severe Dengue and Hospital Acquired Infection. PLoS Negl Trop Dis 2014; 8(4): e2777. DOI:10.1371/journal.pntd.0002777.

15. Halstead S.B, Lucy CS Lum. Assessing the prognosis of dengue-infected patients. F1000 Med Rep 2009; 1: 73. DOI: 10.3410/M1-73.

16. Tantawichien T. Dengue fever and dengue haemorrhagic fever in adults. Southeast Asian J Trop Med Public Health 2015; 46 (Supplement 1).

17. Lee I-K, Liu J-W, Yang KD. Fatal Dengue Hemorrhagic Fever in Adults: Emphasizing the Evolutionary Pre-fatal Clinical and Laboratory Manifestations. PLoS Negl Trop Dis 2012; 6(2): e1532.

DOI:10.1371/journal.pntd.0001532

18. Ong A, Sandar M, Chen MI, Sin LY. Fatal dengue haemorrhagic fever in adults during a dengue epidemic in Singapore. International Journal of Infectious Diseases 2007; 11: 263-67.

19. Yacoub $S$, Wills B. Predicting outcome from dengue. BMC Med. 2014; 12: 147.

DOI: 10.1186/s12916-014-0147-9 PMID: 25259615

20. Wong JGX, Thein TL, Leo Y-S, Pang J, Lye DC. Identifying Adult Dengue Patients at Low Risk for Clinically Significant Bleeding. PLOS ONE 201611 (2): e0148579. DOI: 10.1371/journal.pone.0148579

21. Chamnanchanunt $S$, Kanagaraj $D$, Thanachartwet $V$, Varunee Desakorn V, Rojnuckarin P. Early predictors of clinically significant bleeding in adults with dengue infection. Southeast Asian J Trop Med Public Health 2012; 43 (4): 890-8999. 
22. Díaz-Quijano F A, Martínez-Vega R A, Villar-Centeno L A. Early predictors of haemorrhage in acute febrile syndrome patients from Bucaramanga, Colombia: a dengue endemic area. Singapore Med J. 2008; 49(6): 480.

23. Chandralekha GP, Trikha A. The north Indian dengue outbreak 2006: a retrospective analysis of intensive care unit admissions in a tertiary care hospital. Trans $R$ Soc Trop Med Hygiene 2008; 102: 143-7.

24. Jadhav R, Lohiya S, Agarkhedkar S, Lohakare A, Salunkhe S. Study of dengue fever with special reference to predictors of spontaneous bleeding in dengue infection. $J$ Pharm Biomed Sci. 2015; 05(11): 851-6.

25. Guidelines on Management of DF / DHF in Adults. www.epid.gov.lk/.../guidelines for the management of df and dhf in adults.pdf

26. Comprehensive Guidelines for Prevention and Control of Dengue. www.searo.who.int/entity/vector borne tropical diseases/

27. Souza LJI, Alves JG, Nogueira RM et al. Aminotransferase changes and acute hepatitis in patients with dengue fever: analysis of 1,585 cases. Braz J Infect Dis. 2004; 8(2): 156-63.

DOI: /S1413-86702004000200006

28. Hawkey CJ, Langman MSJ. Non-steroidal anti-inflammatory drugs: overall risks and management. Complementary roles for COX-2 inhibitors and proton pump inhibitors. Gut. 2003; 52(4): 600-8.
29. Russell RI. Non-steroidal anti-inflammatory drugs and gastrointestinal damage - problems and solutions. Postgrad Med J 2001; 77: 82-8.

30. Davies NM. Toxicity of non-steroidal anti-inflammatory drugs in the large intestine. Dis Colon Rectum 1995; 38: 1311. https://doi.org/10.1007/BF02049158

31. Fernando Bessone. Non-steroidal anti-inflammatory drugs: What is the actual risk of liver damage? World $J$ Gastroenterol 2010; 16 (45): 5651-61. DOI: $10.3748 /$ wjg.v16.i45.5651

32. Sisson G, Bjarnason I. Serious upper gastrointestinal complications of NSAIDs and COX-2 selective agents. Inflammopharmacol 2011; 19: 183. DOl.org/10.1007/s10787-011-0085-5

33. Wills $A B$, Oragui $E E$, Stephens $A C$, et al. Coagulation Abnormalities in Dengue Hemorrhagic Fever: Serial Investigations in 167 Vietnamese Children with Dengue Shock Syndrome. Clinical Infectious Diseases 2002; 35(3): 277-85. https://doi.org/10.1086/341410

34. Fernando S, Wijewickrama A, Gomes L, et al. Patterns and causes of liver involvement in acute dengue infection. $B M C$ Infectious Diseases 2016; 16: 319. DOI 10.1186/s12879-016-1656-2 